\title{
Mobile Robot Trajectory Tracking Using Noisy RSS Measurements: An RFID Approach
}

\author{
M. Suruz Miah ${ }^{1}$, Wail Gueaieb ${ }^{2, *}$ \\ School of Electrical Engineering and Computer Science, University of Ottawa, Ontario, K1N 6N5, Canada
}

\begin{abstract}
Most mobile robot navigation techniques that depend on reference RF beacons rely on approximating line-of-sight (LOS) distances between these beacons and the robot. The approximation of LOS is mostly performed using received signal strength (RSS) measurements of signals propagating between the robot and RF beacons. However, to date, relying on RSS measurements for approximating LOS distance remains a significant challenge. Accurate mapping between RSS measurements and LOS distance is almost impossible to achieve in an indoor reverberant environment. In this paper, we design a partially-observed feedback controller for a differential drive mobile robot (DDMR) where the feedback signal is in the form of noisy RSS measurements emitted from radio frequency identification (RFID) tags placed in the environment. The proposed controller does not require an accurate mapping between the LOS distance and the RSS measurements from RFID tags. In addition, it takes into account the robot's actuator (speed) constraints. Unlike many other previously devised solutions, the proposed control scheme does not require the linearization of the nonlinear DDMR model. The performance of this method is demonstrated through both numerical simulations and real-time experiments.
\end{abstract}

Keywords: Mobile robots, Motion control, Optimal control, RFID systems, RF signals.

\section{Introduction}

Controlling nonholonomic mobile robots to track a pre-defined trajectory or reach a desired target point in space is still among the main challenges facing modern robots. Brockett's theorem [1] proves the nonexistence of smooth state-feedbacks for the asymptotic stabilization of fixed configurations. This implies that simplistic linear control schemes would definitely yield significant errors when applied to nonlinear nonholonomic systems.

In recent years, a significant research body has been conducted for solving global stabilization and tracking problems of mobile robots with constrained desired linear and angular velocities. See [2, 3] and some references therein. In most cases, the robot's full-state was assumed to be

\footnotetext{
*Corresponding author

Email address: wgueaieb@eecs. uOttawa.ca (Wail Gueaieb )

${ }^{1}$ Visiting scholar, Machine Intelligence, Robotics, and Mechatronics Laboratory, School of Electrical Engineering and Computer Science (EECS), University of Ottawa, Ontario, Canada

${ }^{2}$ Professor, School of Electrical Engineering and Computer Science (EECS), University of Ottawa, Ontario, Canada 
available to the feedback controllers (see [4, 5, 3], for example). The constraint restriction was eliminated in [6] using an adaptive control technique where the control input is persistently excited. Chwa proposed a solution that is based on two sliding surfaces in polar coordinates for position and heading direction's tracking errors [7]. The satisfactory performance of the controller is clear from the simulations where the mobile robot is able to track any arbitrary trajectory. Nevertheless, this technique does not take into account the actuator constraints. An alternative solution based on backstepping-like feedback linearization was provided in [8]. Despite its satisfactory performance, this technique might not be as effective when applied to nonlinear systems, where states are partially-observed. Recently, a transverse function approach was suggegted to tackle the trajectory tracking problem for nonholonomic mobile robots [9]. A salient feature of this approach is the obtention of feedback laws that unconditionally achieve the practical stabilization of arbitrary reference trajectories, including fixed points and nonadmissible trajectories. However, this is achieved at the cost of the comprehensive tuning of transverse function parameters. A synchronization approach to the problem while maintaining time-varying formations is suggested in [10]. The main idea is to control each robot to track its desired trajectory while synchronizing its motion with that of the other robots in the fleet to preserve the relative kinematics relationships, as required by the formation. Sliding mode control techniques in cooperation with RFID systems have been conducted by numerous researchers in the last decade. See [11] and some references therein. In [11], the RFID tags were placed on the floor in a triangular pattern to estimate the position of the mobile robot. This technique may be suitable for static structured indoor environments but not for dynamically varying ones. Besides trajectory tracking, some researchers contributed to the development of pose estimation paradigms using vision [12, 13, 14]. For such methods to perform well, the noise statistical model has to be known. Furthermore, they have to be embedded in computationally powerful hardware to compensate for their typical high complexity. It is worth pointing out that several closed loop optimal feedback control approaches have been proposed in literatures; however, most of them are focused on solving robotic manipulator problems (not problems of mobile robots), see [15]. Considering full state feedback in model based method for the dynamic of wheeled mobile base with its non-holonomic constraints lead to lack of controllability. In this work, based on using kinematic model of mobile base this problem does not interfere.

It is worth pointing out that several optimal feedback control approaches have been proposed literature but most of them are focused on solving robotic manipulator problems (not problems of mobile robots), see [15], for example, and some references therein.

RFID technology drew the attention of a large body of research on mobile robot localization owing to its wide availability, contactless recognition ability, and affordability [16, 17, 18, 19, 20, 21]. Authors in [22, 23] investigated the application of an RFID system in mobile robot localization by computing the flight time of active beacons' RF signals. Hahnel et al. tried to improve the localization accuracy with a pair of RFID antennas [18]. The phase-difference approach of navigation mobile robots was first introduced by Gueaieb and Miah in [24], where the RFID reader mounted on the robot was customized. The fuzzy logic controller was fed by the phase-difference received at the reader. Nevertheless, this work does not take the robot's timing constraint into account. Moreover, the controller's performance was tested using simulation only. A probabilistic measurement model for the RFID readers was developed for the purpose. Sensor fusion techniques are proposed in [25, 26], where more emphasis is given to solve the robot's localization problem (neither trajectory tracking nor stabilization). Some range-based and bearing-based approaches were also developed [27]. The main idea behind the range-based technique is to trilaterate the robot's position using some known reference points and the esti- 
mated distances at those points in the environment. Distances can be estimated from either RSS measurements or time-based methods. Although the use of the time of flight (ToF) [28] and time difference of arrival (TDoA) measurements [29] were investigated by some, RSS remains generally the feature of choice for indoor positioning. This is due to the fact that RSS measurements can be obtained relatively effortlessly and inexpensively. In addition, no extra hardware (e.g., ultrasonic or infra-red) is needed for network-centric localization [30]. On the other hand, bearing-based schemes use the direction of arrival (DoA) of a target. These schemes usually require multiple range sensors in order to be better suited for mobile robot applications [27].

This manuscript contributes to the efforts of developing trajectory tracking systems for indoor mobile robots while resolving some of the aforementioned limitations. The work described herein is pioneered by mounting an RFID reader on a mobile robot and placing customized RFID tags in the 3-D space of an indoor operating environment [31]. The main contribution of this work is the development of a trajectory tracking controller using an optimal feedback control law without a prior knowledge of the noise statistical model of the RSS measurements of the RF signals transmitted by the RFID tags. To the best of our knowledge, this is the first attempt of using RSS measurements in a feedback control system for such an application.

The rest of the paper is outlined as follows. Some preliminary mathematical conventions and properties are provided in section 2 . Section 3 illustrates the high level architecture of the proposed mobile robot trajectory tracking system. The mathematical formulation of the proposed tracking problem is given in section 4 . Section 5 describes the optimal measurement feedback control law for a differential drive mobile robot. A thorough evaluation of the proposed algorithm along with numerical simulation results are presented in section 6 Section 7 demonstrates the performance of the proposed controller through a real-time experiment. Finally, conclusions and some future research avenues are drawn in section 8 .

\section{Preliminaries}

In the rest of the paper, small and capital bold letters will be used to denote vectors and matrices, respectively. Scalars will be denoted by non-bold letters. The 2-norm and scalar product are defined by

$$
\|\mathbf{x}\| \equiv\left[\sum_{i=1}^{n}\left|x_{i}\right|^{2}\right]^{1 / 2} \text { and }(\mathbf{x} \cdot \mathbf{y}) \equiv \mathbf{x}^{T} \mathbf{y} \equiv \sum_{i=1}^{n} x_{i} y_{i}
$$

respectively, for vectors $\mathbf{x}, \mathbf{y} \in \mathbb{R}^{n}$ and positive $n$. For matrices $\mathbf{X}, \mathbf{Y} \in \mathbb{R}^{m \times n}$, these quatities are given by

$$
\|\mathbf{X}\| \equiv\left[\sum_{i=1}^{m} \sum_{j=1}^{n}\left|x_{i, j}\right|^{2}\right]^{1 / 2} \text { and }(\mathbf{X} \cdot \mathbf{Y}) \equiv \operatorname{Tr}\left[\mathbf{X}^{T} \mathbf{Y}\right] \equiv \operatorname{Tr}\left[\mathbf{X} \mathbf{Y}^{T}\right],
$$

respectively, where $\operatorname{Tr}[\cdot]$ denotes the trace of matrix [·]. Clearly, $\operatorname{Tr}\left[\mathbf{X}^{T} \mathbf{X}\right]=\|\mathbf{X}\|^{2}$.

If the function $J: \mathbb{R}^{n} \rightarrow \mathbb{R}$ is differentiable at $\mathbf{x} \in \mathbb{R}^{n}$, then for any $\mathbf{v} \in \mathbb{R}^{n}, d J(\mathbf{x} ; \mathbf{v})$ denotes the Gateaux (directional) derivative in the direction of $\boldsymbol{v}$, which is given by

$$
d J(\mathbf{x} ; \mathbf{v})=\mathbf{v}^{T} \nabla J=\lim _{\varepsilon \rightarrow 0} \frac{J(\mathbf{x}+\varepsilon \mathbf{v})-J(\mathbf{x})}{\varepsilon},
$$


However, if $J: \mathbb{R}^{m \times n} \rightarrow \mathbb{R}$, then for any $\mathbf{X}, \mathbf{V} \in \mathbb{R}^{m \times n}$, the directional derivative in the direction of $\mathbf{V}$ is defined by

$$
d J(\mathbf{X} ; \mathbf{V})=\operatorname{Tr}\left[\mathbf{V}^{T} \nabla J\right]=\lim _{\varepsilon \rightarrow 0} \frac{J(\mathbf{X}+\varepsilon \mathbf{V})-J(\mathbf{X})}{\varepsilon} .
$$

For any bounded interval $I \equiv\left[t_{0}, t_{f}\right], C\left(I, \mathbb{R}^{n}\right)$ denotes the class of all continuous functions on $I$ taking values in $\mathbb{R}^{n}$. Let $p \in[1, \infty)$ and any finite time interval $I$, we use $\mathcal{L}_{p}\left(I, \mathbb{R}^{n}\right)$ to denote the set of Lebesgue measurable functions $\{\mathbf{f}\}$ defined on the measurable set $I$ and taking values in $\mathbb{R}^{n}$ whose norms are $p$-th power integrable [32, 33] i.e.,

$$
L_{p}(\mathbf{f})=\left(\int_{t_{0}}^{t_{f}}\|\mathbf{f}\|^{p} d t\right)^{1 / p}<\infty,
$$

where $L_{p}(\mathbf{f})$ denotes the $p$-th norm of the function $\mathbf{f}$. For $p=\infty, \mathcal{L}_{\infty}\left(I, R^{n}\right)$ denotes the space of Lebesgue measurable functions $\{\mathbf{f}\}$ defined on $I$ and taking values in $\mathbb{R}^{n}$ satisfying

$$
\operatorname{ess-\operatorname {sup}\{ \| \mathbf {f}(t)\| ,t\in I\} <\infty .}
$$

Similarly, $\mathcal{L}_{p}^{\ell o c}\left([0, \infty), \mathbb{R}^{n}\right)$ are locally convex topological vector spaces of $p$-th power locally integrable functions containing the spaces $\mathcal{L}_{p}\left(I, \mathbb{R}^{n}\right)$.

\section{High-Level System Architecture}

A high level setup of the proposed tracking system with four RFID tags, Tag1, Tag2, Tag3, and Tag4, attached to the ceiling of an indoor space (office, for instance), is depicted in Fig. 1 . In this configuration, the robot's desired trajectory on the ground is defined in continuous time, $\mathbf{q}^{d}(t)$ with $\mathbf{q}^{d}\left(t_{0}\right)$ and $\mathbf{q}^{d}\left(t_{f}\right)$ being the initial and final poses, respectively. For instance, if a mobile robot is provided with the list of 16-bit tag IDs, 0xFFF9, 0xFFF2, 0xFFF5, and 0xFFF4, then it is supposed to continuously read these tag IDs and their corresponding RSS values through an RFID reader mounted on it [31]. Based on the tags' RSS measurements, optimal control actions are then generated for its actuators to track the desired trajectory, $\mathbf{q}^{d}(t)$. It is worth mentioning that although Fig. 1 shows a 4-tag system, the controller proposed herein is applicable to any number of tags. Flowchart of the proposed trajectory tracking algorithm is presented in Fig. 2 ,

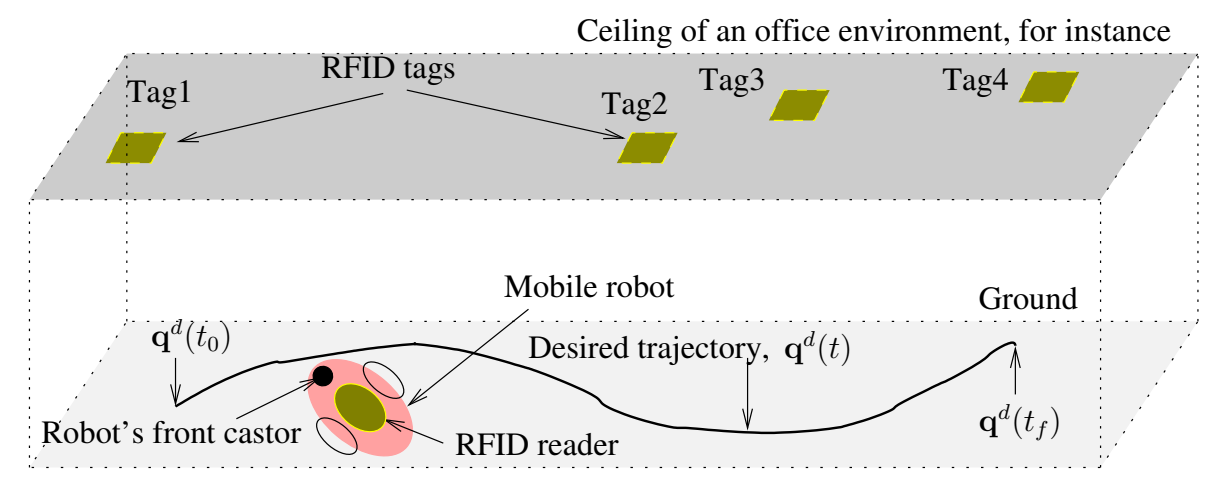

Figure 1: High level system architecture of the proposed tracking system.

where the robot's tracking control strategy is divided into two cascaded phases. The first phase 
(see (0), (1), (2) in Fig. 2) provides the optimal time-varying control gain, $\mathbf{K}^{o}(t)$, using off-line computation. The second phase (see (3), (4), (5) in Fig. 2) takes into account the actual noisy RSS measurements from RFID tags for the robot to generate optimal control actions using the optimal feedback gain computed in the first phase. In the following, we provide a detailed description of how these optimal control actions are generated for the robot to track its desired trajectory.

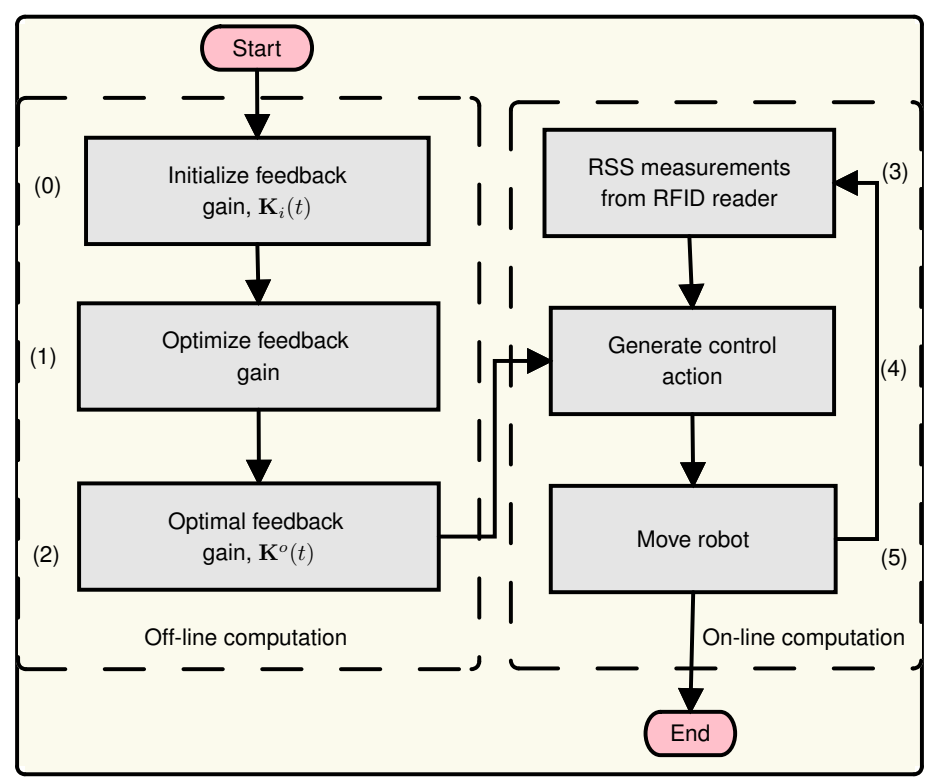

Figure 2: Flowchart of the proposed trajectory tracking algorithm.

\section{Problem Formulation}

A kinematic representation of a differential-drive mobile robot is shown in Fig. 3. Let $(x, y)$ and $\theta$ be the position and the heading angle of a robot with respect to a ground-fixed inertial reference frame X-Y. The rotational velocities of the robot's left and right wheels are characterized by the sole (scaler) axis angular velocities $u_{L}$ and $u_{R}$, respectively; under the assumption that the front castor wheel is passive and simply provides necessary support for its balance. The robot's position $(x, y)$ is the coordinates of the midpoint of the wheelbase of length $l$ connecting the two lateral wheels along their axis.

Let $t_{0}$ and $t_{f}$ be the initial and final instants of the robot's travel time, respectively, and let $I \equiv\left[t_{0}, t_{f}\right]$. At any time $t \in I$, the robot kinematics is governed by

$$
\left[\begin{array}{c}
\dot{x}(t) \\
\dot{y}(t) \\
\dot{\theta}(t)
\end{array}\right]=\frac{r}{2}\left[\begin{array}{cc}
\cos \theta(t) & \cos \theta(t) \\
\sin \theta(t) & \sin \theta(t) \\
\frac{2}{l} & -\frac{2}{l}
\end{array}\right]\left[\begin{array}{l}
u_{R}(t) \\
u_{L}(t)
\end{array}\right],
$$

where $r$ is the radius of each wheel. A compact form of (1) can be written as

$$
\dot{\mathbf{q}}(t)=\underset{5}{\mathbf{f}[\mathbf{q}(t), \mathbf{u}(t)]}=\frac{r}{2} \mathbf{B}[\mathbf{q}(t)] \mathbf{u}(t),
$$




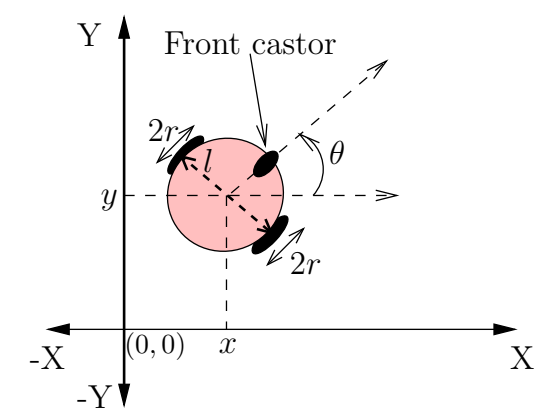

Figure 3: Kinematic model of a differential-drive mobile robot.

where the robot's configuration $\mathbf{q}(t) \equiv\left[\begin{array}{lll}x(t) & y(t) & \theta(t)\end{array}\right]^{T} \in \mathbb{R}^{2} \times \mathbb{S}^{1}$, its control input vector $\mathbf{u}(t) \equiv\left[u_{R}(t) u_{L}(t)\right]^{T} \in \mathbb{R}^{2}$,

$$
\mathbf{B}[\mathbf{q}(t)]=\left[\begin{array}{cc}
\cos \theta(t) & \cos \theta(t) \\
\sin \theta(t) & \sin \theta(t) \\
\frac{2}{l} & -\frac{2}{l}
\end{array}\right],
$$

and $\mathbf{f}: \mathbb{R}^{2} \times \mathbb{S}^{1} \times \mathbb{R}^{2} \rightarrow \mathbb{R}^{2} \times \mathbb{S}^{1}$. However, due to the speed limits of the wheels, the inputs are contrained as

$$
\left|u_{L}(t)\right| \leq u_{L}^{\max } \text { and }\left|u_{R}(t)\right| \leq u_{R}^{\max }, t \in I .
$$

In other words, $\mathbf{u}(t)$ must be chosen from a set of admissible speeds, $\mathcal{U}_{a d}$, i.e., $\mathbf{u}(t) \in \mathcal{U}_{a d}$. Note that a DDMR is an underactuated system in which the number of control inputs is less than the number of state variables to be controlled. In this example, there are two control inputs $\left(u_{R}(t), u_{L}(t)\right)$ and three state variables $(x(t), y(t), \theta(t))$ to be controlled. In addition, a DDMR is also a nonholonomic system with the nonholonomic contraint given by

$$
\dot{x} \sin \theta-\dot{y} \cos \theta=0,
$$

which ensures the wheel's non-slip movement in the lateral direction [34, ch. 12].

The RSS measurements are modeled as

$$
\mathbf{z}(t)=\hat{\mathbf{h}}[\mathbf{q}(t)]+\zeta(t),
$$

where $\mathbf{z}(t) \in \mathbb{R}^{s}$ is the RSS measurement vector (in $\mathrm{dBm}$ ) from $s$ RFID tags in the environment and $\zeta(t) \in \mathbb{R}^{s}$ is an unknown dynamic noise associated to it. The nonlinear measurement function $\hat{\mathbf{h}}^{T}[\mathbf{q}(t)]=\left[\hat{h}_{1}[\mathbf{q}(t)] \ldots \hat{h}_{s}[\mathbf{q}(t)]\right]$ of $\left[5\right.$ is given by $\mathbf{h}: \mathbb{R}^{2} \times \mathbb{S}^{1} \rightarrow \mathbb{R}^{s}$ with

$$
\hat{h}_{j}[\mathbf{q}(t)]=\alpha e^{\beta \hat{d}_{j}}, \text { for } j=1, \ldots, s,
$$

where $\alpha$ and $\beta$ are unknown parameters which are dependent on the operating environment. $\hat{d}_{j}$ of (6) is the estimated Euclidean distance between the robot's measured position $(x, y)$ and the known $j$-th RFID tag position $\mathbf{q}_{t}^{j}=\left[x_{t}^{j} y_{t}^{j} z_{t}^{j}\right]^{T}$. Thus,

$$
\hat{d}_{j}=\sqrt{\left(x-x_{t}^{j}\right)^{2}+\left(y-y_{t}^{j}\right)^{2}+\left(z_{t}^{j}\right)^{2}}, \text { for } j=1, \ldots, s .
$$


We now define the feedback control $\mathbf{u}(t)$ as

$$
\mathbf{u}(t)=\mathbf{K}(t) \mathbf{z}(t)
$$

subject to (3), where $\mathbf{K}(t) \in \mathcal{K}_{a d} \subset \mathbb{R}^{2 \times s}, t \in I$, is the feedback control gain for the system (2). Substituting (7) in (2) and using the measurement model (5), the robot's feedback system can be formulated as

$$
\begin{gathered}
\dot{\mathbf{q}}(t)=\hat{\mathbf{f}}[\mathbf{q}(t), \mathbf{K}(t)]+\hat{\mathbf{g}}[\mathbf{q}(t), \mathbf{K}(t)], \text { where } \\
\hat{\mathbf{f}}[\mathbf{q}(t), \mathbf{K}(t)] \equiv \frac{r}{2} \mathbf{B}[\mathbf{q}(t)] \mathbf{K}(t) \hat{\mathbf{h}}[\mathbf{q}(t)] \text { and } \hat{\mathbf{g}}[\mathbf{q}(t), \mathbf{K}(t)] \equiv \frac{r}{2} \mathbf{B}(\mathbf{q}(t)) \mathbf{K}(t) \boldsymbol{\zeta}(t) .
\end{gathered}
$$

The block diagram of the proposed feedback system is shown in Fig. 4 . The reference trajectory block is to synthesize feasible nominal trajectory, $\mathbf{q}^{d}(t), t \in I$. The optimal controller block takes the tracking error and the tags' noisy RSS measurements as inputs to generate optimal control signal $\mathbf{u}(t)$ which is dispatched to the robot's actuators. Since feedback control gain $\mathbf{K}(t)$ in (7) needs to be optimized to generate optimal values of $\mathbf{u}(t)$, the current feedback control problem is reduced to an optimization problem.

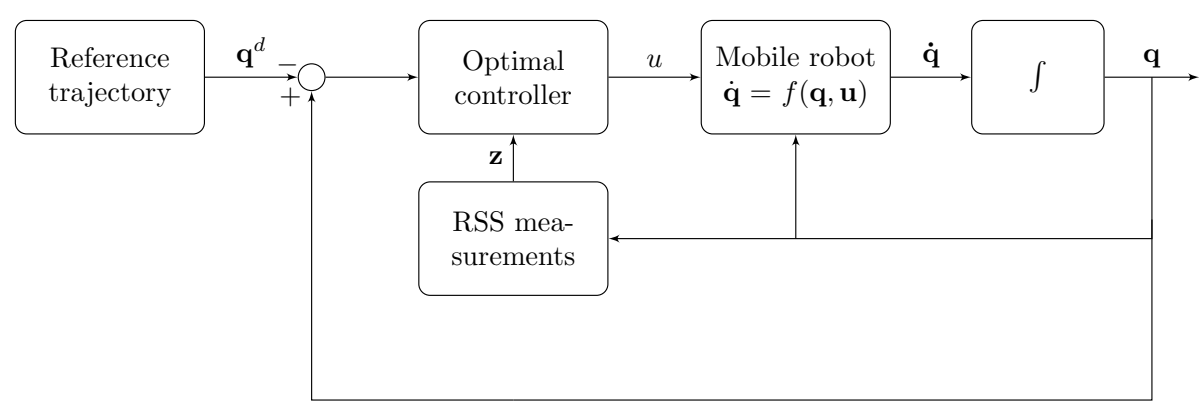

Figure 4: Feedback structure of the proposed trajectory tracking method.

Let $\mathbf{q}^{d}(t)=\left[x^{d}(t) y^{d}(t) \theta^{d}(t)\right]^{T}$ be the desired trajectory that the robot is supposed to track and $e(t)=\sqrt{\left[x^{d}(t)-x(t)\right]^{2}+\left[y^{d}(t)-y(t)\right]^{2}}$ denote its position tracking error, for $t \in I$. The objective is to find the optimal control input $\mathbf{u}(t) \in \mathcal{U}_{a d}$ that generates the trajectory $\mathbf{q}(t) \in Q$ while minimizing the total position tracking error, $\mathscr{E}$, given by

$$
\mathscr{E}=\int_{t_{0}}^{t_{f}} e(t) d t .
$$

Given the robot's kinematic model (2), its nonholonimic constraint (4), and for any $\zeta(t)$, the problem can be stated as follows:

$$
\inf _{\left\{\mathbf{q} \in Q, \mathbf{u} \in \mathcal{U}_{a d}\right\}}[\mathscr{E}] .
$$

Although not explicitly stated, this goal implicitly imposes the optimization of the robot orientation $\theta(t)$ since it is coupled with the robot position (see model (2)). This point will be clearer in the next section. 


\section{Optimal Feedback Control Law}

The purpose of this section is to study the proposed optimal feedback control law for determining the control inputs (i.e., speeds) which are applied to the robot's actuators for tracking a certain desired trajectory in its workspace. For that, we introduce the following assumptions:

(A1): since the input $\mathbf{u}(t) \in \mathcal{U}_{a d}$ is the piecewise constant bounded function; that is, $\mathbf{u}(t) \in$ $\mathcal{L}_{\infty}\left([0, \infty), \mathbb{R}^{2}\right)$, the set $\mathcal{K} \subset \mathbb{R}^{2 \times s}$ must be a closed bounded convex set and $\mathcal{K}_{a d} \equiv\left\{\mathbf{K}(t) \in \mathcal{L}_{\infty}\left([0, \infty), \mathbb{R}^{2 \times s}\right): \mathbf{K}(t) \in \mathcal{K}\right\}$. (A2): the noise vector of the measurement model $[5], \zeta:[0, \infty) \rightarrow \mathbb{R}^{s}$, can be any measurable stochastic process taking values from the closed (Euclidean) ball $\mathcal{B}\left(\bar{\zeta}, r^{\prime}\right)$ defined by

$$
\mathcal{B}\left(\bar{\zeta}, r^{\prime}\right)=\left\{\zeta(t) \in \mathbb{R}^{s}:\|\zeta(t)-\bar{\zeta}\| \leq r^{\prime}\right\},
$$

where $r^{\prime}>0$ is the radius of the noise associated with the RSS measurements and $\bar{\zeta}$ is the mean of $\zeta(t)$, for $t \in I$.

In order to find the optimal control input $\mathbf{u}(t) \in \mathcal{U}_{a d}$ that generate the trajectory $\mathbf{q}(t), t \in I$, let us define the cost functional as

$$
\begin{gathered}
J(\mathbf{K}, \zeta)=\phi\left[t_{f}, \mathbf{q}\left(t_{f}\right)\right]+\int_{t_{0}}^{t_{f}} \ell[t, \mathbf{q}(t)] d t, \text { where } \\
\phi\left[t_{f}, \mathbf{q}\left(t_{f}\right)\right]=\frac{1}{2}\left[\mathbf{q}\left(t_{f}\right)-\mathbf{q}^{d}\left(t_{f}\right)\right]^{T} \mathbf{P}\left(t_{f}\right)\left[\mathbf{q}\left(t_{f}\right)-\mathbf{q}^{d}\left(t_{f}\right)\right], \\
\ell[t, \mathbf{q}(t)]=\frac{1}{2}\left[\mathbf{q}(t)-\mathbf{q}^{d}(t)\right]^{T} \mathbf{Q}(t)\left[\mathbf{q}(t)-\mathbf{q}^{d}(t)\right] .
\end{gathered}
$$

where $\mathbf{P}\left(t_{f}\right), \mathbf{Q}(t) \in \mathbb{R}^{3 \times 3}$ are symmetric positive definite matrices that indicate the relative importance of the error components along $\mathbb{R}^{2} \times \mathbb{S}^{1}$. If the robot's purpose is to reach a desired target point in its environment, then the weight matrix $\mathbf{P}\left(t_{f}\right)$ must be higher than $\mathbf{Q}(t)$. However, the opposite is true if the robot's main mission is to track a desired trajectory. The performance index $J(\mathbf{K}, \boldsymbol{\zeta})$ depends on the feedback control gain matrix $\mathbf{K}(t)$ and the measurement noise $\zeta(t)$ through the state variable $\mathbf{q}(t)$ as it is clear from the feedback system (8). Note that the cost function (11) is similar to the performance measure defined in Bolza type problem, where $\phi\left[t_{f}, \mathbf{q}\left(t_{f}\right)\right]$ represents the terminal cost and $\ell[t, \mathbf{q}(t)]$ represents the running cost [35].

To solve for the optimal trajectory using the feedback system (8) that minimizes the objective functional (11), we need to derive the necessary conditions of optimality. These necessary conditions are most readily found if the integrand of the cost functional (11) is recast in terms of Hamiltonian $\mathcal{H}: I \times \mathbb{R}^{2} \times \mathbb{S}^{1} \times \mathbb{R}^{3} \times \mathbb{R}^{2 \times s} \longrightarrow \mathbb{R}$, which is expressed by

$$
\mathcal{H}[\mathbf{q}(t), \psi(t), \mathbf{K}(t)]=\psi^{T}[\hat{\mathbf{f}}[\mathbf{q}(t), \mathbf{K}(t)]+\hat{\mathbf{g}}[\mathbf{q}(t), \mathbf{K}(t)]]+\ell(t, \mathbf{q}(t)),
$$

where $\psi(t) \in \mathbb{R}^{3}, t \in I$, is a vector of Lagrange multipliers whose elements are the costates of the system [35]. Before deriving the necessary conditions of optimality, we present the following Lemma for the robot's feedback model (8).

Lemma 1 (Solutions of feedback system). Given assumptions (A1)-(A2) and the feedback system (8), which is defined over the finite time horizon of $I$, then for every initial condition $\mathbf{q}(0) \in$ 
$\mathbb{R}^{2} \times \mathbb{S}^{1}$, and feedback control gain $\mathbf{K}(t) \in \mathcal{K}_{\text {ad }}$, the system 8 , has a unique absolutely continuous solution $\mathbf{q}(t) \in C\left(I, \mathbb{R}^{2} \times \mathbb{S}^{1}\right)$. Furthermore, the solution set

$$
Q \equiv\left\{\mathbf{q}(t) \equiv \mathbf{q}[t, \mathbf{K}(t), \zeta(t)] \in C\left(I, \mathbb{R}^{2} \times \mathbb{S}^{1}\right): \mathbf{K}(t) \in \mathcal{K}_{a d}\right\}
$$

is a bounded subset of $C\left(I, \mathbb{R}^{2} \times \mathbb{S}^{1}\right)$.

The proof of this lemma is classical and follows from a similar technique in [36, p. 89]. Thus, if $\mathbf{q}_{0} \equiv \mathbf{q}\left(t_{0}\right)$ denotes the robot's pose at time $t_{0}$, the actual trajectory of the robot can be described by

$$
\mathbf{q}(t)=\mathbf{q}_{0}+\int_{t_{0}}^{t}\{\hat{\mathbf{f}}[\mathbf{q}(\tau), \mathbf{K}(\tau)]+\hat{\mathbf{g}}[\mathbf{q}(\tau), \mathbf{K}(\tau)]\} d \tau, \quad \mathbf{q}(t) \in Q, t \in I .
$$

Theorem 1 (Necessary conditions of optimality). Consider the feedback system (8) satisfying lemma 1 The optimal trajectory $\mathbf{q}^{\circ}(t), t \in I$ can be obtained if there exists an optimal feedback control gain $\mathbf{K}^{o}(t) \in \mathcal{K}_{a d}$ and an optimal multiplier $\psi^{o}(t) \in C\left(I, \mathbb{R}^{3}\right)$ such that the triple $\left\{\mathbf{q}^{o}(t), \boldsymbol{\psi}^{o}(t), \mathbf{K}^{o}(t)\right\}$ satisfies the following necessary conditions:

$$
\begin{gathered}
\mathcal{H}\left[\mathbf{q}^{o}(t), \psi^{o}(t), \mathbf{K}(t)\right] \geq \mathcal{H}\left[\mathbf{q}^{o}(t), \psi^{o}(t), \mathbf{K}^{o}(t)\right], \mathbf{K}(t) \in \mathcal{K}, t \in I, \\
\dot{\mathbf{q}}^{o}=\frac{\partial \mathcal{H}}{\partial \boldsymbol{\psi}}\left[\mathbf{q}^{o}(t), \psi^{o}(t), \mathbf{K}^{\mathbf{o}}(t)\right], \mathbf{q}^{\mathbf{o}}\left(t_{0}\right)=\mathbf{q}_{\mathbf{0}}, t \in I, \\
\dot{\psi}^{o}=-\frac{\partial \mathcal{H}}{\partial \mathbf{q}}\left[\mathbf{q}^{o}(t), \psi^{o}(t), \mathbf{K}^{o}(t)\right], \psi^{o}\left(t_{f}\right)=\frac{\partial \phi}{\partial \mathbf{q}}\left[t_{f}, \mathbf{q}\left(t_{f}\right)\right] .
\end{gathered}
$$

Proof. Let $\mathbf{q}(t) \equiv \mathbf{q}[t, \mathbf{K}(t), \zeta(t)]$ be the solution of the feedback system $(8)$, with the cost functional (11) for any choice of $\mathbf{K}(t) \in \mathcal{K}_{a d}$. For simplicity of the proof, and without loss of generality, assume that the noise vector $\zeta(t) \equiv \zeta_{c}$ is fixed. Since $\mathbf{K}^{o}(t)$ is optimal with the associated trajectory $\mathbf{q}^{o}(t)$, it is clear that

$$
J\left[\mathbf{K}^{o}(t), \zeta_{c}\right] \leq J\left[\mathbf{K}(t), \zeta_{c}\right], \quad \forall \mathbf{K}(t) \in \mathcal{K}_{a d}, t \in I .
$$

Suppressing the variable $t$ for clarity and for any $\varepsilon \in[0,1]$, we define $\mathbf{K}^{\varepsilon}=\mathbf{K}^{o}+\varepsilon\left(\mathbf{K}-\mathbf{K}^{o}\right)$. Since $\mathcal{K}$ is a closed convex set, $\mathcal{K}_{a d}$ is also a closed convex subset of $\mathcal{L}_{\infty}\left(I, \mathbb{R}^{2 \times s}\right)$ and therefore, $\mathbf{K}^{\varepsilon} \in \mathcal{K}_{a d}$. Thus, $J\left(\mathbf{K}^{o}, \zeta_{c}\right) \leq J\left(\mathbf{K}^{\varepsilon}, \boldsymbol{\zeta}_{c}\right)$, which yields

$$
\operatorname{Tr}\left[\left(\mathbf{K}-\mathbf{K}^{o}\right)^{T} d J\left(\mathbf{K}^{o}, \boldsymbol{\zeta}_{c}\right)\right] \geq 0,
$$

where $d J\left(\mathbf{K}^{o}, \boldsymbol{\zeta}_{c}\right)$ denotes the Gateaux (directional) derivative of $J$ evaluated at $\mathbf{K}=\mathbf{K}^{o}$ in the direction of $\mathbf{K}-\mathbf{K}^{o}$.

Let $\mathbf{q}^{\varepsilon}$ be the solution of the feedback system (8) corresponding to the gain $\mathbf{K}^{\varepsilon}$ with the same initial state $\mathbf{q}^{\varepsilon}\left(t_{0}\right)=\mathbf{q}_{0}$. It is easy to veryify that

$$
\lim _{\varepsilon \rightarrow 0} \mathbf{K}^{\varepsilon}(t)=\mathbf{K}^{o}(t) \text {, and } \quad \lim _{\varepsilon \rightarrow 0} \mathbf{q}^{\varepsilon}(t)=\mathbf{q}^{o}(t) .
$$

Hence, the state trajectories $\mathbf{q}^{\varepsilon}(t)$ and $\mathbf{q}^{o}(t)$ become defined as

$$
\dot{\mathbf{q}}^{\varepsilon}=\hat{\mathbf{f}}\left(\mathbf{q}^{\varepsilon}, \mathbf{K}^{\varepsilon}\right)+\hat{\mathbf{g}}\left(\mathbf{q}^{\varepsilon}, \mathbf{K}^{\varepsilon}\right) \text {, and } \dot{\mathbf{q}}^{o}=\hat{\mathbf{f}}\left(\mathbf{q}^{o}, \mathbf{K}^{o}\right)+\hat{\mathbf{f}}\left(\mathbf{q}^{o}, \mathbf{K}^{o}\right),
$$


with $\mathbf{q}^{\varepsilon}\left(t_{0}\right)=\mathbf{q}^{o}\left(t_{0}\right)=\mathbf{q}_{0}$ and $t \in I$. Defining

$$
\eta(t) \equiv \lim _{\varepsilon \rightarrow 0}\left(\frac{\mathbf{q}^{\varepsilon}(t)-\mathbf{q}^{o}(t)}{\varepsilon}\right),
$$

it follows that $\boldsymbol{\eta}(t)$ must satisfy the following initial value problem

$$
\dot{\boldsymbol{\eta}}=\left[\frac{\partial \hat{\mathbf{f}}}{\partial \mathbf{q}}\left(\mathbf{q}^{o}, \mathbf{K}^{o}\right)+\frac{\partial \hat{\mathbf{g}}}{\partial \mathbf{q}}\left(\mathbf{q}^{o}, \mathbf{K}^{o}\right)\right] \boldsymbol{\eta}+\hat{\mathbf{f}}\left(\mathbf{q}^{o}, \mathbf{K}-\mathbf{K}^{o}\right)+\hat{\mathbf{g}}\left(\mathbf{q}^{o}, \mathbf{K}-\mathbf{K}^{o}\right),
$$

with $\boldsymbol{\eta}\left(t_{0}\right)=0$. Equation 18$)$ is linear and non-homogeneous with $\hat{\mathbf{f}}\left(\mathbf{q}^{o}, \mathbf{K}-\mathbf{K}^{o}\right)+\hat{\mathbf{g}}\left(\mathbf{q}^{o}, \mathbf{K}-\mathbf{K}^{o}\right)$ being the driving force. As a result, it has a continuous solution $\boldsymbol{\eta}(t) \in C\left(I, \mathbb{R}^{3}\right)$, which is continuously dependent on $\hat{\mathbf{f}}\left(\mathbf{q}^{o}, \mathbf{K}-\mathbf{K}^{o}\right)+\hat{\mathbf{g}}\left(\mathbf{q}^{o}, \mathbf{K}-\mathbf{K}^{o}\right)$.

By definition of Gateaux (directional) derivative we can derive the following expression

$$
\begin{aligned}
& \operatorname{Tr}\left[\left(\mathbf{K}-\mathbf{K}^{o}\right)^{T} d J\left(\mathbf{K}^{o}, \boldsymbol{\zeta}_{c}\right)\right]=\lim _{\varepsilon \rightarrow 0} \frac{J\left(\mathbf{K}^{o}+\varepsilon\left(\mathbf{K}-\mathbf{K}^{o}\right), \boldsymbol{\zeta}_{c}\right)-J\left(\mathbf{K}^{o}, \boldsymbol{\zeta}_{c}\right)}{\varepsilon} \\
& =\boldsymbol{\eta}^{T}(t) \frac{\partial \phi}{\partial \mathbf{q}}\left[t_{f}, \mathbf{q}\left(t_{f}\right)\right]+\int_{t_{0}}^{t_{f}} \boldsymbol{\eta}^{T}(t) \frac{\partial \ell}{\partial \mathbf{q}}[t, \mathbf{q}(t)] d t .
\end{aligned}
$$

Hence, inequality (17) yields

$$
\boldsymbol{\eta}^{T}(t) \frac{\partial \phi}{\partial \mathbf{q}}\left[t_{f}, \mathbf{q}\left(t_{f}\right)\right]+\int_{t_{0}}^{t_{f}} \boldsymbol{\eta}^{T}(t) \frac{\partial \ell}{\partial \mathbf{q}}[t, \mathbf{q}(t)] d t \geq 0
$$

Since $\boldsymbol{\eta}(t)$ of the variational equation $(18)$ is continuously dependent on $\hat{\mathbf{f}}\left(\mathbf{q}^{o}, \mathbf{K}-\mathbf{K}^{o}\right)+\hat{\mathbf{g}}\left(\mathbf{q}^{o}, \mathbf{K}-\right.$ $\mathbf{K}^{o}$ ), the map

$$
\hat{\mathbf{f}}\left(\mathbf{q}^{o}, \mathbf{K}-\mathbf{K}^{o}\right)+\hat{\mathbf{g}}\left(\mathbf{q}^{o}, \mathbf{K}-\mathbf{K}^{o}\right) \longmapsto \boldsymbol{\eta}(t), \quad t \in I
$$

has to be continuous from $\mathcal{L}_{1}\left(I, \mathbb{R}^{3}\right)$ to $C\left(I, \mathbb{R}^{3}\right)$ [36, p. 260]. The map

$$
\boldsymbol{\eta}(t) \longmapsto \boldsymbol{\eta}^{T}(t) \frac{\partial \phi}{\partial \mathbf{q}}\left[t_{f}, \mathbf{q}\left(t_{f}\right)\right]+\int_{t_{0}}^{t_{f}} \boldsymbol{\eta}^{T}(t) \frac{\partial \ell}{\partial \mathbf{q}}[t, \mathbf{q}(t)] d t
$$

is then a continuous linear functional on $C\left(I, \mathbb{R}^{3}\right)$. Thus, the composition map

$$
\hat{\mathbf{f}}\left(\mathbf{q}^{o}, \mathbf{K}-\mathbf{K}^{o}\right)+\hat{\mathbf{g}}\left(\mathbf{q}^{o}, \mathbf{K}-\mathbf{K}^{o}\right) \longmapsto \boldsymbol{\eta}^{T}(t) \frac{\partial \phi}{\partial \mathbf{q}}\left[t_{f}, \mathbf{q}\left(t_{f}\right)\right]+\int_{t_{0}}^{t_{f}} \boldsymbol{\eta}^{T}(t) \frac{\partial \ell}{\partial \mathbf{q}}[t, \mathbf{q}(t)] d t
$$

is a continuous linear functional on $\mathcal{L}_{1}\left(I, \mathbb{R}^{3}\right)$, where

$$
\left[\hat{\mathbf{f}}\left(\mathbf{q}^{o}, \mathbf{K}-\mathbf{K}^{o}\right)+\hat{\mathbf{g}}\left(\mathbf{q}^{o}, \mathbf{K}-\mathbf{K}^{o}\right)\right] \in \mathcal{L}_{1}\left(I, \mathbb{R}^{3}\right)
$$

. Therefore, by the Riesz representation theorem or by the duality [33] between $\mathcal{L}_{1}\left(I, \mathbb{R}^{3}\right)$ and $\mathcal{L}_{\infty}\left(I, \mathbb{R}^{3}\right)$, we may conclude that there exists an element $\psi^{o} \in \mathcal{L}_{\infty}\left(I, \mathbb{R}^{3}\right)$ such that

$$
\begin{aligned}
& \operatorname{Tr}\left[\left(\mathbf{K}-\mathbf{K}^{o}\right)^{T} d J\left(\mathbf{K}^{o}, \zeta_{c}\right)\right]=\boldsymbol{\eta}^{T} \frac{\partial \phi}{\partial \mathbf{q}}\left[t_{f}, \mathbf{q}\left(t_{f}\right)\right]+\int_{t_{0}}^{t_{f}} \boldsymbol{\eta}^{T} \frac{\partial \ell}{\partial \mathbf{q}}[t, \mathbf{q}(t)] d t \\
& =\int_{t_{0}}^{t_{f}}\left(\boldsymbol{\psi}^{o}\right)^{T}\left[\hat{\mathbf{f}}\left(\mathbf{q}^{o}, \mathbf{K}-\mathbf{K}^{o}\right)+\hat{\mathbf{g}}\left(\mathbf{q}^{o}, \mathbf{K}-\mathbf{K}^{o}\right)\right] d t
\end{aligned}
$$


It follows from inequality [19] that $\forall \mathbf{K} \in \mathcal{K}_{a d}$,

$$
\int_{t_{0}}^{t_{f}}\left(\psi^{o}\right)^{T}\left[\hat{\mathbf{f}}\left(\mathbf{q}^{o}, \mathbf{K}-\mathbf{K}^{o}\right)+\hat{\mathbf{g}}\left(\mathbf{q}^{o}, \mathbf{K}-\mathbf{K}^{o}\right)\right] d t \geq 0 .
$$

Using the variational equation (18) and (20)

$$
\begin{aligned}
& \boldsymbol{\eta}^{T}(t) \frac{\partial \phi}{\partial \mathbf{q}}\left[t_{f}, \mathbf{q}\left(t_{f}\right)\right]+\int_{t_{0}}^{t_{f}} \boldsymbol{\eta}^{T}(t) \frac{\partial \ell}{\partial \mathbf{q}}[t, \mathbf{q}(t)] d t= \\
& \int_{t_{0}}^{t_{f}}\left(\boldsymbol{\psi}^{o}\right)^{T}\left[\dot{\boldsymbol{\eta}}-\left\{\frac{\partial \hat{\mathbf{f}}}{\partial \mathbf{q}}\left(\mathbf{q}^{o}, \mathbf{K}^{o}\right)+\frac{\partial \hat{\mathbf{g}}}{\partial \mathbf{q}}\left(\mathbf{q}^{o}, \mathbf{K}^{o}\right)\right\} \boldsymbol{\eta}\right] d t .
\end{aligned}
$$

Integrating by parts and since $\boldsymbol{\eta}\left(t_{0}\right)=0$, expression (22) can now be written as

$$
\begin{gathered}
\boldsymbol{\eta}^{T}(t) \frac{\partial \phi}{\partial \mathbf{q}}\left[t_{f}, \mathbf{q}\left(t_{f}\right)\right]+\int_{t_{0}}^{t_{f}} \boldsymbol{\eta}^{T}(t) \frac{\partial \ell}{\partial \mathbf{q}}[t, \mathbf{q}(t)] d t=\boldsymbol{\eta}^{T}\left(t_{f}\right) \boldsymbol{\psi}^{o}\left(t_{f}\right)+ \\
\int_{t_{0}}^{t_{f}} \boldsymbol{\eta}^{T}\left[-\dot{\boldsymbol{\psi}}^{o}-\left\{\left(\frac{\partial \hat{\mathbf{f}}}{\partial \mathbf{q}}\left(\mathbf{q}^{o}, \mathbf{K}^{o}\right)\right)^{T}+\left(\frac{\partial \hat{\mathbf{g}}}{\partial \mathbf{q}}\left(\mathbf{q}^{o}, \mathbf{K}^{o}\right)\right)^{T}\right\} \boldsymbol{\psi}^{o}\right] d t,
\end{gathered}
$$

and so

$$
\begin{aligned}
\dot{\psi}^{o} & =-\left\{\left(\frac{\partial \hat{\mathbf{f}}}{\partial \mathbf{q}}\left(\mathbf{q}^{o}, \mathbf{K}^{o}\right)\right)^{T}+\left(\frac{\partial \hat{\mathbf{g}}}{\partial \mathbf{q}}\left(\mathbf{q}^{o}, \mathbf{K}^{o}\right)\right)^{T}\right\} \psi^{o} \\
& -\frac{\partial \ell}{\partial \mathbf{q}}\left[t, \mathbf{q}^{o}(t)\right], \quad \psi^{o}\left(t_{f}\right)=\frac{\partial \phi}{\partial \mathbf{q}}\left[t_{f}, \mathbf{q}\left(t_{f}\right)\right] .
\end{aligned}
$$

This costate dynamics equation is linear along the optimal trajectories. Thus, the necessary conditions of optimality are given by the integral inequality (21), the costate dynamics (24), and the state equation (8). In other words, the choice of $\mathbf{K} \in \mathcal{K}_{a d}$ determines the optimality conditions [21], 24], and (8).

Reformulating the optimality condition 21 as

$$
\int_{t_{0}}^{t_{f}}\left(\psi^{o}\right)^{T}\left[\hat{\mathbf{f}}\left(\mathbf{q}^{o}, \mathbf{K}\right)+\hat{\mathbf{g}}\left(\mathbf{q}^{o}, \mathbf{K}\right)\right] d t \geq \int_{t_{0}}^{t_{f}}\left(\psi^{o}\right)^{T}\left[\hat{\mathbf{f}}\left(\mathbf{q}^{o}, \mathbf{K}^{o}\right)+\hat{\mathbf{g}}\left(\mathbf{q}^{o}, \mathbf{K}^{o}\right)\right] d t,
$$

$\forall \mathbf{K} \in \mathcal{K}_{a d}$, it becomes easy to derive the following point-wise inequality [36]

$$
\left(\psi^{o}\right)^{T}\left[\hat{\mathbf{f}}\left(\mathbf{q}^{o}, \mathbf{K}\right)+\hat{\mathbf{g}}\left(\mathbf{q}^{o}, \mathbf{K}\right)\right] \geq\left(\psi^{o}\right)^{T}\left[\hat{\mathbf{f}}\left(\mathbf{q}^{o}, \mathbf{K}^{o}\right)+\hat{\mathbf{g}}\left(\mathbf{q}^{o}, \mathbf{K}^{o}\right)\right],
$$

$\forall \mathbf{K} \in \mathcal{K}_{a d}$. Adding the term $\ell\left[t, \mathbf{q}^{o}(t)\right]$ in both sides, yields

$$
\begin{aligned}
\left(\psi^{o}\right)^{T} & {\left[\hat{\mathbf{f}}\left(\mathbf{q}^{o}, \mathbf{K}\right)+\hat{\mathbf{g}}\left(\mathbf{q}^{o}, \mathbf{K}\right)\right]+\ell\left[t, \mathbf{q}^{o}(t)\right] \geq } \\
& \left(\boldsymbol{\psi}^{o}\right)^{T}\left[\hat{\mathbf{f}}\left(\mathbf{q}^{o}, \mathbf{K}^{o}\right)+\hat{\mathbf{g}}\left(\mathbf{q}^{o}, \mathbf{K}^{o}\right)\right]+\ell\left[t, \mathbf{q}^{o}(t)\right],
\end{aligned}
$$

which gives the following Hamiltonian inequality

$$
\mathcal{H}\left[\mathbf{q}^{o}(t), \psi^{o}(t), \mathbf{K}(t)\right] \geq \mathcal{H}\left[\mathbf{q}^{o}(t), \psi^{o}(t), \mathbf{K}^{o}(t)\right] .
$$


This is the same as inequality (14) stated in the Theorem. Differentiating $\mathcal{H}[\cdot]$ in $[12]$ with respect to the costate variable $\psi$, we get

$$
\frac{\partial \mathcal{H}}{\partial \psi}\left[\mathbf{q}^{o}(t), \psi^{o}(t), \mathbf{K}^{o}(t)\right]=\hat{\mathbf{f}}\left[\mathbf{q}^{o}(t), \mathbf{K}^{o}(t)\right]+\hat{\mathbf{g}}\left[\mathbf{q}^{o}(t), \mathbf{K}^{o}(t)\right],
$$

which leads to the state equation

$$
\dot{\mathbf{q}}^{o}=\frac{\partial \mathcal{H}}{\partial \psi}\left[\mathbf{q}^{o}(t), \psi^{o}(t), \mathbf{K}^{o}(t)\right], \quad \mathbf{q}^{o}\left(t_{0}\right)=\mathbf{q}_{0},
$$

as defined in (15).

Differentiating the Hamiltonian with respect to the state variable $\mathbf{q}$ yields

$$
\begin{aligned}
& \frac{\partial \mathcal{H}}{\partial \mathbf{q}}\left[\mathbf{q}^{o}(t), \psi^{o}(t), \mathbf{K}^{\mathbf{o}}(t)\right]=\left\{\left(\frac{\partial \hat{\mathbf{f}}}{\partial \mathbf{q}}\left(\mathbf{q}^{o}, \mathbf{K}^{o}\right)\right)^{T}+\left(\frac{\partial \hat{\mathbf{g}}}{\partial \mathbf{q}}\left(\mathbf{q}^{o}, \mathbf{K}^{o}\right)\right)^{T}\right\} \psi^{o}+\frac{\partial \ell}{\partial \mathbf{q}}\left[t, \mathbf{q}^{o}(t)\right] \\
& =-\dot{\psi}^{o} .
\end{aligned}
$$

Hence, the costate dynamics 24) can be expressed in terms of the Hamiltonian as

$$
\dot{\psi}^{o}=-\frac{\partial \mathcal{H}}{\partial \mathbf{q}}\left[\mathbf{q}^{o}(t), \psi^{o}(t), \mathbf{K}^{o}(t)\right], \psi^{o}\left(t_{f}\right)=\frac{\partial \phi}{\partial \mathbf{q}}\left[t_{f}, \mathbf{q}\left(t_{f}\right)\right],
$$

which is condition 16 .

Theorem 1 states that there exists a feedback control gain $\mathbf{K}^{o} \in \mathcal{K}_{a d}$ for the robot to determine optimal control inputs for its actuator. In order to solve for $\mathbf{K}^{o}$, we express the gradient of the Hamiltonian defined in (12) as

$$
\frac{\partial \mathcal{H}}{\partial \mathbf{K}}=\frac{r}{2}\left[(\mathbf{B}(\mathbf{q}))^{T} \psi \hat{\mathbf{h}}^{T}(\mathbf{q})+(\mathbf{B}(\mathbf{q}))^{T} \psi \zeta^{T}\right]
$$

The gradient $\mathcal{H}_{\mathbf{K}} \equiv \frac{\partial \mathcal{H}}{\partial \mathbf{K}}$ indicates the direction of convergence for the optimal trajectory of 8 . Having defined $\mathbf{q}(t)$ in (13) and $\psi(t)$ in (16) for $t \in I$, the optimal $\mathbf{K}$ can be found by setting the gradient 277 to be zero, i.e.,

$$
\frac{r}{2}\left[(\mathbf{B}(\mathbf{q}))^{T} \psi \hat{\mathbf{h}}^{T}(\mathbf{q})+(\mathbf{B}(\mathbf{q}))^{T} \psi \zeta^{T}\right]=0 .
$$

Note that expression $(28)$ is independent of the gain matrix $\mathbf{K}$. Hence, the problem boils down to finding $\mathbf{K}(t), t \in I$, such that the robot's equation of motion (13) and the costate trajectory from (16), satisfy (28). The optimal feedback control gain $\mathbf{K}^{o}$ can be determined by satisfying the Hamiltonian inequality (14). In other words, $\mathbf{K}$ is to be adaptively tuned to minimize the robot's tracking error.

Corollary 1 (Adapting the gain K). Consider the robot's feedback system (8) defined over the time horizon I. Adapting the gain $\mathbf{K}$ according to the following offline update rule

$$
\mathbf{K}^{\text {new }}=\mathbf{K}^{\text {old }}-\epsilon \mathcal{H}_{\mathbf{K}}, \text { for } 0<\epsilon<1
$$

satisfies the Hamiltonian inequality (14) and, hence, guarantees the converge of the robot's trajectory towards its target. 
Proof. Let

$$
\mathbf{K}^{*}=\mathbf{K}-\epsilon \mathcal{H}_{\mathbf{K}},
$$

for some $\mathbf{K} \in \mathbb{R}^{2 \times s}$ and $0<\epsilon<1$. The corresponding Hamiltonian is

$$
\mathcal{H}\left(\mathbf{q}^{*}, \boldsymbol{\psi}^{*}, \mathbf{K}^{*}\right)=\mathcal{H}\left(\mathbf{q}^{*}, \psi^{*}, \mathbf{K}-\epsilon \mathcal{H}_{\mathbf{K}}\right),
$$

where $\mathbf{q}^{*}$ and $\boldsymbol{\psi}^{*}$ are the robot's pose and the costate variable corresponding to $\mathbf{K}^{*}$. Taking Taylor series expansion of the right hand side about $\mathbf{K}$, we get

$$
\mathcal{H}\left(\mathbf{q}^{*}, \boldsymbol{\psi}^{*}, \mathbf{K}^{*}\right)=\mathcal{H}\left(\mathbf{q}^{*}, \boldsymbol{\psi}^{*}, \mathbf{K}\right)+\operatorname{Tr}\left[\mathcal{H}_{\mathbf{K}}^{T}\left(\mathbf{K}^{*}-\mathbf{K}\right)\right]+\mathcal{O}(\varepsilon) .
$$

Neglecting the higher order terms of the above expression yields

$$
\begin{aligned}
\mathcal{H}\left(\mathbf{q}^{*}, \boldsymbol{\psi}^{*}, \mathbf{K}^{*}\right) & \equiv \mathcal{H}\left(\mathbf{q}^{*}, \boldsymbol{\psi}^{*}, \mathbf{K}\right)-\epsilon \operatorname{Tr}\left[\mathcal{H}_{\mathbf{K}}^{T} \mathcal{H}_{\mathbf{K}}\right] \\
& =\mathcal{H}\left(\mathbf{q}^{*}, \boldsymbol{\psi}^{*}, \mathbf{K}\right)-\epsilon\left\|\mathcal{H}_{\mathbf{K}}\right\|^{2}<\mathcal{H}\left(\mathbf{q}^{*}, \boldsymbol{\psi}^{*}, \mathbf{K}\right)
\end{aligned}
$$

since $\epsilon\left\|\mathcal{H}_{\mathbf{K}}\right\|^{2}>0$. Hence, update rule (30) guarantees that the Hamiltonian $\mathcal{H}$ is monotonically decreasing, which proves that the adaptation law (29) eventually leads to the optimal control gain $\mathbf{K}^{o}$.

In the following, we numerically solve for the gain $\mathbf{K}$ such that $[28]$ is satisfied, aggregating the components described earlier.

Let $\mathbf{K}_{i} \equiv \mathbf{K}_{i}(t), t \in I$, be the gain at the $i$-th iteration of the optimization procedure.

Step 0 (initialization): Subdivide the time interval $I \equiv\left[t_{0}, t_{f}\right]$ into $N$ subintervals. Assume a piecewise-contant $\mathbf{K}_{\mathbf{i}}(t)=\mathbf{K}_{\mathbf{i}}\left(t_{k}\right), t \in\left[t_{k}, t_{k+1}\right]$, for $k=0, \ldots, N-1$, and choose any $\zeta \in \mathcal{B}\left(\bar{\zeta}, r^{\prime}\right)$. Find the optimal gain $\mathbf{K}^{o}$ by repeating Steps $1-5$ until the stopping criterion in Step 5 is met.

Step 1: Integrate the robot's feedback system (8) as in (13) with $\mathbf{K} \equiv \mathbf{K}_{\mathbf{i}}(t)$.

Step 2: Solve costate equation (16) backward for $\psi_{i}$.

Step 3: Define the Hamiltonian $\mathcal{H}\left(\mathbf{q}_{i}, \boldsymbol{\psi}_{i}, \mathbf{K}_{i}\right)$ as in (12).

Step 4: Compute the cost function $J\left(\mathbf{K}_{i}, \zeta\right)$ using (11), the gradients of the Hamiltonian $\mathcal{H}_{\mathbf{K}}$ using 27, and its corresponding intergrated norm $\int_{t_{0}}^{t_{f}}\left\|\mathcal{H}_{\mathbf{K}}\right\|^{2} d t$.

Step 5: If $J\left(\mathbf{K}_{i}, \zeta\right) \leq \delta_{1}$ or $\int_{t_{0}}^{t_{f}}\left\|\mathcal{H}_{\mathbf{K}}\right\|^{2} d t \leq \delta_{2}$, for pre-defined small positive tolerance constants $\delta_{1}$ and $\delta_{2}$, then $\mathbf{K}_{\mathbf{i}}$ is regarded close enough to its nominal optimal value, and so the algorithm is halted.

Otherwise, use the following update rule to adjust the piecewise-constant feedback control gain:

$$
\mathbf{K}_{i+1}\left(t_{k}\right)=\mathbf{K}_{i}\left(t_{k}\right)-\epsilon \mathcal{H}_{\mathbf{K}\left(t_{k}\right)}+\lambda \Delta \mathbf{K}_{i}\left(t_{k}\right), \Delta \mathbf{K}_{i}\left(t_{k}\right)=\mathbf{K}_{i}\left(t_{k}\right)-\mathbf{K}_{i-1}\left(t_{k}\right)
$$

for $k=0, \ldots, N-1$, where $\epsilon$ and $\lambda$ are the step size and the momentum constant (for faster convergence), respectively.

\section{Simulation Results}

We now illustrate the performance of the proposed trajectory tracking system using a circular shaped differential drive virtual mobile robot with a wheel base of $l=30 \mathrm{~cm}$ and a wheel radius of $r=8.25 \mathrm{~cm}$. The rotational speeds of the right and left wheels are constrained by $\left|u_{R}\right| \leq$ 
Table 1: Sample RSS measurements from XBee Pro module.

\begin{tabular}{|l|c|c|c|c|c|c|c|}
\hline Distance $(d) \mathrm{m}$ & 3.00 & 3.13 & 3.50 & 4.04 & 4.69 & 5.41 & 6.18 \\
\hline RSS $(\mathrm{dBm})$ & -47 & -48 & -51 & -62 & -54 & -64 & -69 \\
\hline
\end{tabular}

$u_{R}^{\max }=12.1 \mathrm{rad} \cdot \mathrm{s}^{-1}$, and $\left|u_{L}\right| \leq u_{L}^{\max }=12.1 \mathrm{rad} \cdot \mathrm{s}^{-1}$. The size of the virtual test environment is $25 \times 20 \times 3 \mathrm{~m}$ high. A mesh of 9 RFID tags is mounted on the ceiling. The orthogonal projection of the tags' locations on the ground are shown in Fig.7(a) Note that these locations are unknown to the robot. The performance metric adopted in the current work is the tracking error between the robot's desired and actual trajectories over the time period of $I \equiv[0,60] \mathrm{s}$, as defined in 9 . Initially, the feedback control gain $\boldsymbol{K}$ is chosen as:

$$
\boldsymbol{K}(t)=10^{-6}\left[\begin{array}{llll}
1 & 1 & 1 & 1 \\
1 & 1 & 1 & 1
\end{array}\right], \quad t \in I .
$$

The sampling time period is set to $0.1 \mathrm{~s}$. The weighting matrices of the cost function (11) are chosen as $\mathbf{P}\left(t_{f}\right)=\operatorname{diag}(0.5,0.5,1)$ and $\mathbf{Q}(t)=\operatorname{diag}(1,1,2), \forall t \in I$. Hence, trajectory tracking is regarded twice as important as just reaching the final destination.

The grid of RFID tags attached to the ceiling with one tag every $3 \mathrm{~m}$, ensured that the robot always receives RF signals whose RSS values are within the range of the sampled data (see Fig. 6(a)]. The size of the grid (i.e., number of tags) and the inter-tag distance depend on the size of the robot's workspace and the communication range between the tags and the reader. In practical applications, these parameters should be chosen as to guarantee that, at any given time, the robot can receive "meaningful" RSS values from a minimum number of tags ( 9 in this application). In this context, a "meaningful" RSS value is one that is within the range used in the calibration process (Fig. 6(a) in this case). This is not to be confused with trilateration-based localization methods, because as explained in the previous sections, they are not used in our work.

\subsection{Modelling RSS Measurements and Noise}

To make the controller's simulation as realistic as possible, the RSS signals were experimentally measured by emulating the RFID system using an XBee Pro RF module and a MakeController board (Fig. 5] [31]. The RFID emulation was necessary since till the time this manuscript was written, none of the commercially available RFID readers had the capability of providing the RSS value of the RF signal. A theoretical design of a modified RFID architecture that supports this feature is presented in [37, 38].

For that, the XBee Pro RF module (emulating the RFID tag) was attached on a 3-m high ceiling in our research laboratory while the MakeController board (emulating the RFID reader) was mounted on a dummy robot. Then, the robot was manually placed in various positions on the ground. For each position, the average RSS measurements transmitted by the XBee module is recorded as received by the MakeController board. Table 1 shows some sample RSS measurements logged in this experiment and the measurements are plotted in Fig. 6(b) Since the purpose of this exercise is to use these measurements as semi-ground truth for the noise-free signal model, precautions were taken to reduce the effect of reverberations from the ground and the far-end walls. Fitting the data to model (6) yielded the parameter values $\alpha=-35.5$ and $\beta=0.1071$. 
In order to match real-world scenarios where the robot's workspace may be populated with reverberant obstacles, such as walls, metal file cabinets, appliances, etc., this model was superimposed with an exaggerated Gaussian noise $\zeta(t)$ (see the measurement model (5)) defined by

$$
\operatorname{Pr}[\zeta(t)]=\frac{1}{\sqrt{2 \pi \sigma^{2}}} \exp \left[-\frac{\{\zeta(t)-\bar{\zeta}\}^{2}}{2 \sigma^{2}}\right],
$$

with a mean $\bar{\zeta}=-30 \mathrm{dBm}$ and a standard deviation $\sigma=50 \mathrm{dBm}$. This yielded a signal-tonoise ratio of $-179.45 \mathrm{dBm}$. This weak signal-to-noise ratio was adopted on purpose to better demonstrate later the performance of the proposed controller. Fig. 6(a) shows the "noise-free" and noisy RSS signals obtained. Fitting the resultant noisy RSS signal again to model (6) led to the parameter values of $\alpha=-60$ and $\beta=0.2$. This model of the noisy signal was used in the following controller's simulations.

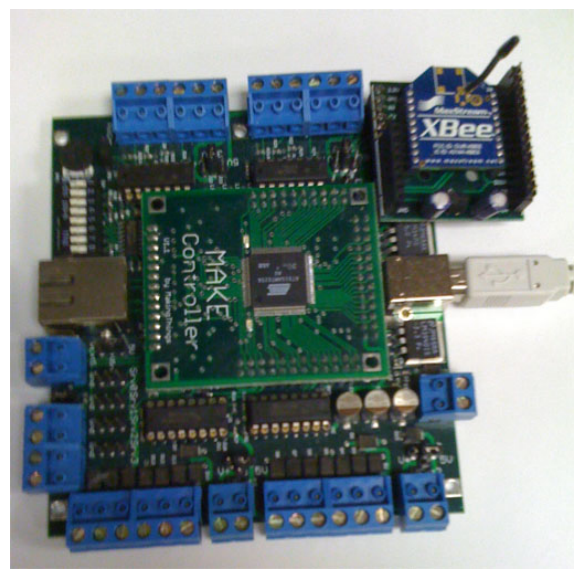

(a)

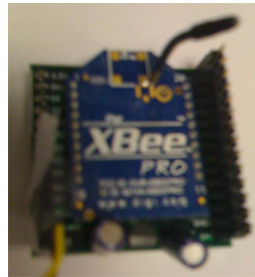

(b)

Figure 5: (a) MakeController board, and (b) XBee Pro RF module.

\subsection{Tracking a Curvilinear Trajectory}

The purpose of this test scenario is to study the robot's tracking ability along a more complex path. To do that, a desired trajectory was defined as $x^{d}(t)=(6 / 60) t-3, y^{d}(t)=3 \sin \left[1.2 x^{d}(t)\right]$, and $\theta^{d}(t)=\tan ^{-1}\left[d y^{d} / d x^{d}\right]=\tan ^{-1}\left\{3.6 \cos \left[1.2 x^{d}(t)\right]\right\}$. The robot is initially placed at $(-2.5,1.5) \mathrm{m}$ with an initial orientation of $0^{\circ}$. The results are demonstrated in Fig. 7. Despite the large initial error of $0.53 \mathrm{~m}$, the robot converged to within $0.1 \mathrm{~m}$ of the target trajectory in about $6 \mathrm{~s}$ (Fig. 7(b)]. Since then, it maintained a tracking error of less than $0.15 \mathrm{~m}$ till the end of the simulation. The percentile plot in Fig. 7(b) shows that the tracking error was less than $0.1 \mathrm{~m}$ for $80 \%$ of the time. This could have been better if not for the large initial error, which was set so on purpose to test the robot's convergence speed. The total distance traveled by the robot in this simulation is $16.4 \mathrm{~m}$. The average tracking error throughout the whole trajectory was recorded to be $0.08 \mathrm{~m}$. It is important to point out here that one of the salient features of the proposed navigation system is that its tracking error is non-cumulative (independent of the total traveled distance). This can be clearly noticed from the tracking error plot in Fig. 7(b) 


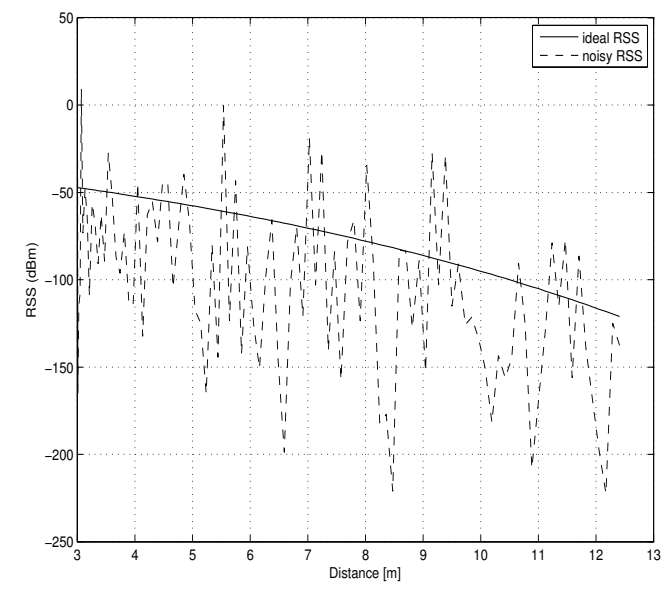

(a)

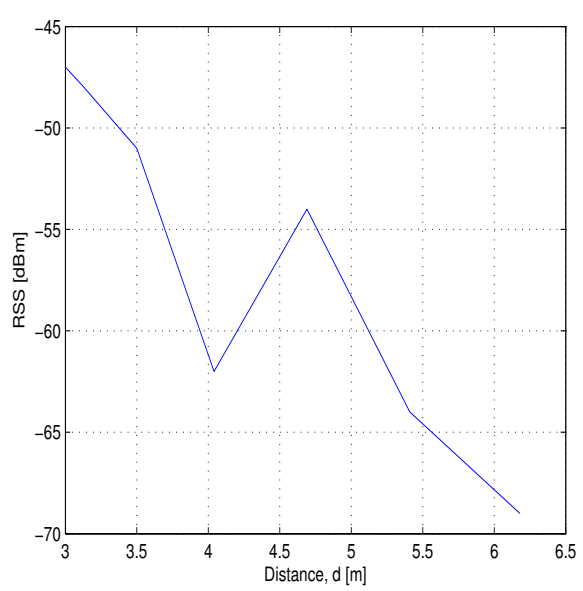

(b)

Figure 6: RSS vs. distance (a) noise model considered for the simulation and (b) RSS measurements from XBee Pro module.

\section{Experimental Results}

An experiment is conducted using the Sputnik 3 DMR with a wheelbase of $l=26 \mathrm{~cm}$ and a wheel radius of $r=8.25 \mathrm{~cm}$. The experiment took space in a laboratory of $10 \times 9 \times 3 \mathrm{~m} \mathrm{high}$. A top-view of the workspace floor plan is depicted in Fig. 8. The robot is set to follow a U-shaped trajectory divided into three segments, $A=7.5 \mathrm{~m}, B=4.0 \mathrm{~m}$, and $C=5.0 \mathrm{~m}$, in a total time $t_{f}=60 \mathrm{~s}$. Four XBee modules are deployed at 3D positions $(1.7,5.0,0.7) \mathrm{m},(-1.5,4.9,0.7) \mathrm{m}$, $(-1.5,2.1,0.7) \mathrm{m}$, and $(1.0,2.6,0.7) \mathrm{m}$. The MakeController board emulating the RFID reader is mounted on top of the robot. The XBee modules transmit information wirelessly to the MakeController board through a laptop which acts as a "hub" in this case. As a matter of fact, no data processing is run by the laptop and all the controller's computations are performed on the robot's unique microcontroller.

The robot's real-time performance in tracking the U-shaped trajectory is revealed in Fig. 9 (a) where the hollow and solid arrows indicate the robot's initial and final poses, respectively. The corresponding tracking error is shown in Fig. 9(b). Note that the robot had overshoot at the sharp corners as it is clear from the error spikes at times $t \approx 26 \mathrm{~s}$ and $t \approx 41 \mathrm{~s}$ (see Fig. 9(b)). However, it came back to track the trajectory, as expected. The robot's tracking performance is quite satisfactory in the sense that $90 \%$ of the time the error is less than $10 \mathrm{~cm}$ (see percentile plot of Fig. 9(b)]. Snapshots of this experiment are provided in Fig. 10, where Figs. $10(\mathrm{a}), 10(\mathrm{~d}),[10(\mathrm{e})$ $10(\mathrm{~g})$, and $10(\mathrm{~h})$ 10(j) reveal the navigation performance on segments $\mathrm{A}, \mathrm{B}$, and $\mathrm{C}$, respectively. It is worth noticing that a line of sight exists between the MakeController board and each of the four XBee modules when the robot navigates along segment $\mathrm{A}$. This becomes no longer the case along most of segment $\mathrm{B}$ and all of segment $\mathrm{C}$ due to the work benches in the middle of the lab (see Fig. 8). Nevertheless, the robot was able to track the whole trajectory with a tracking error

${ }^{3}$ http://www.drrobot.com/ 


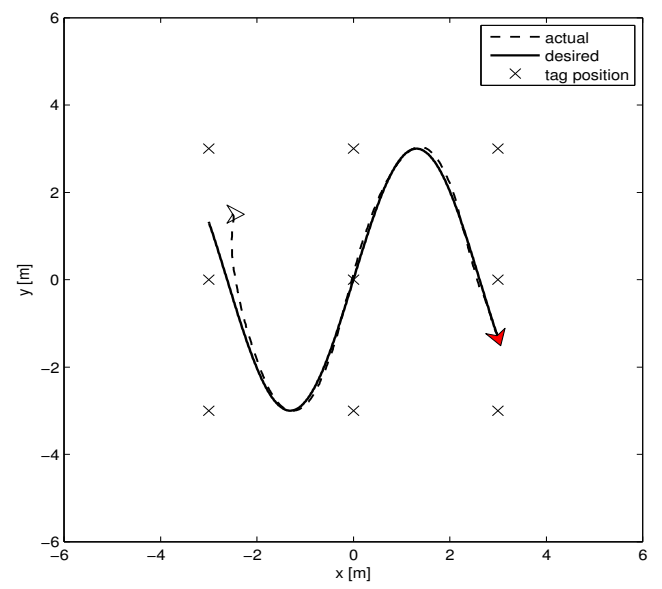

(a)

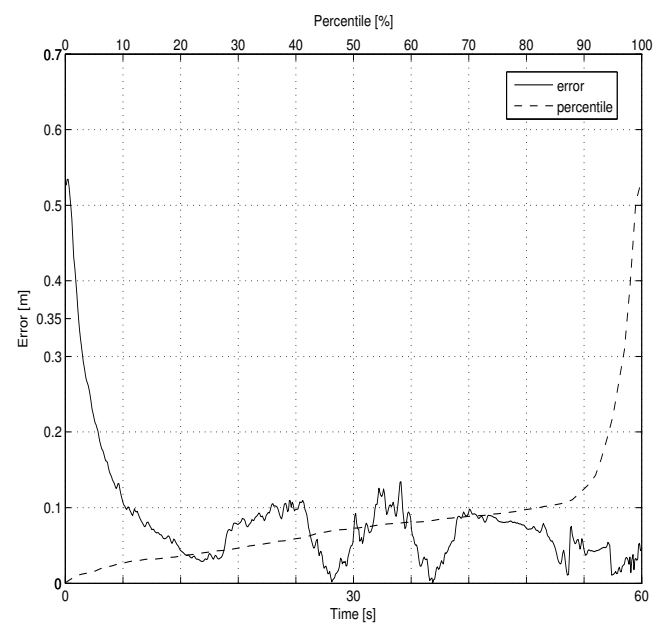

(b)

Figure 7: Controller's performance in following a curvilinear trajectory: (a) robot's trajectory and (b) tracking error.

of no more than $10 \mathrm{~cm}$ from the wheelbase center point. An overshoot is noticed around each corner before the robot converges back to its target trajectory in about $1 \mathrm{~s}$. The robot stopped about $7 \mathrm{~cm}$ away from the desired end point (see Fig. 10(j). This is not surprising since the experiment was treated by the proposed controller as a trajectory tracking problem rather than fixed-point stabilization.

In summary, the simulation and experimental results demonstrate a satisfactory accuracy level of the suggested control scheme despite the weak signal-to-noise ratio characterizing the RSS signals received by the RFID reader. It is worth mentioning that this controller is also able to stabilize the robot at a fixed configuration by simply tuning the weight matrices $\left(\mathbf{P}\left(t_{f}\right)\right.$ and $\left.\mathbf{Q}(t)\right)$ of the cost function (11). This is in contrast to many recent RFID-based techniques which usually tackle the localization problem only [21, 39, 16]. The localization accuracy reported there in is in the range of $0.1-0.5 \mathrm{~m}$, despite neglecting the effect of reverberations and low signal-to-noise ratios.

It is clear from the experimental results that the robot is still able to track the desired trajectory with a satisfactory tracking error $(\approx 10 \mathrm{~cm})$ even it loses its line of sight with one or all of the XBee modules. This is regarded as one of the most appealing features in the proposed technique. Yet, we believe that the tracking error achieved in this work can be further reduced by adopting different types of cheap and computationally-simple sensors (i.e., sonar, infra-red, laser range finder, etc.) in conjunction with the RFID system.

\section{Conclusion}

The rising prominence of guiding a mobile robot in many real-life indoor robotic applications necessitates the development of suitable navigation systems to track certain desired trajectories. Due to the ubiquity of such systems, a novel non-visual trajectory tracking system is developed here for this purpose. It is based on processing the RSS signals of RFID tags mounted in the 


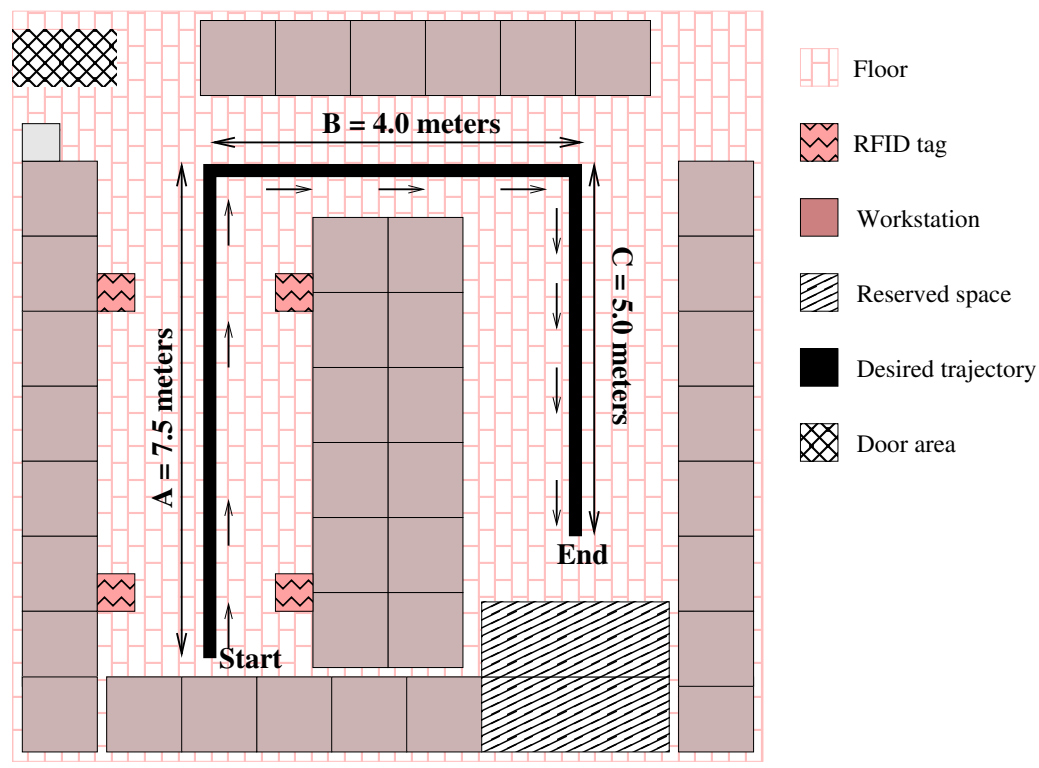

Figure 8: Experimental setup: floor plan of the robot's workspace.

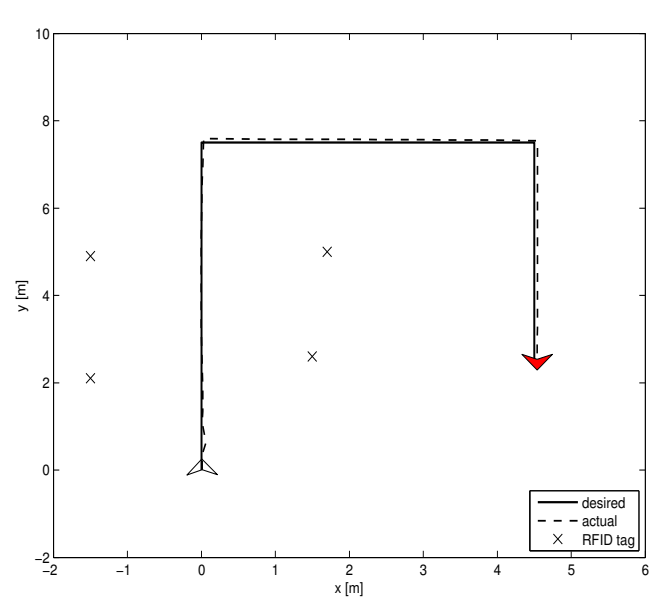

(a)

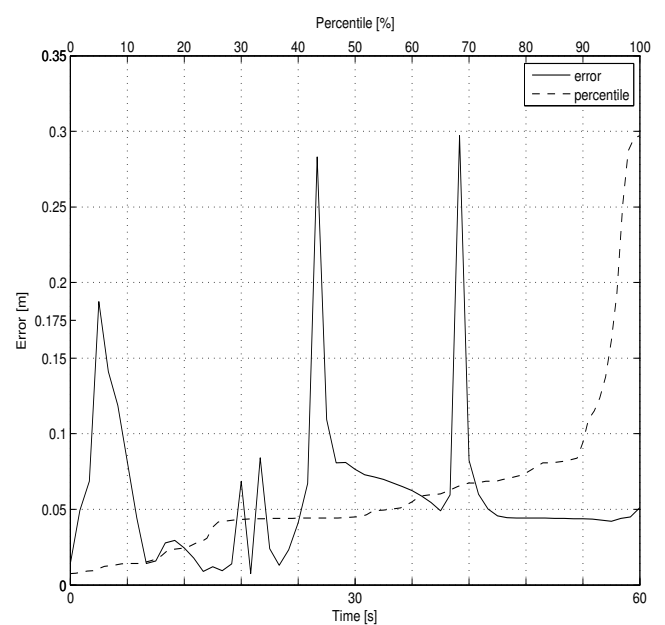

(b)

Figure 9: Robot's real-time performance:(a) actual vs. desired trajectory and (b) tracking error 


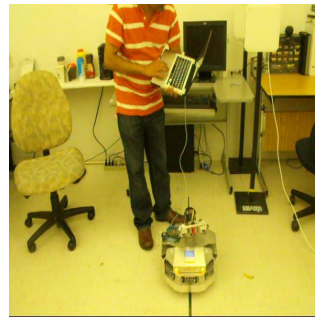

(a)

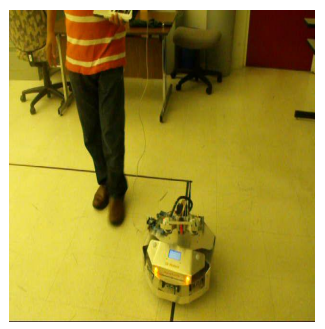

(e)

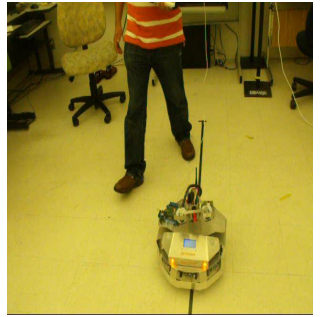

(b)

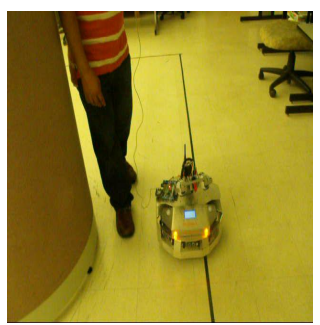

(f)

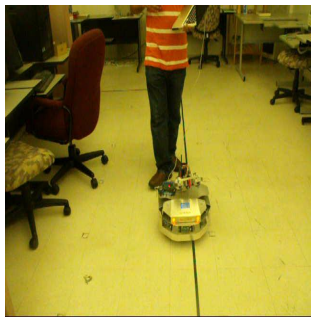

(i)

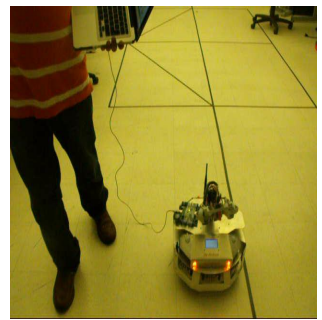

(c)

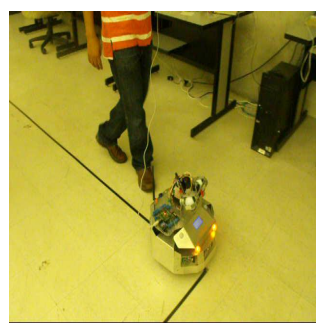

(g)

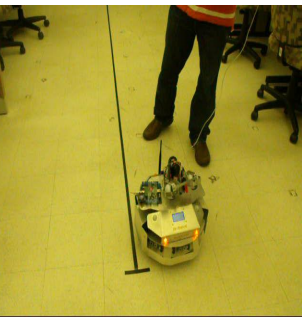

(j)

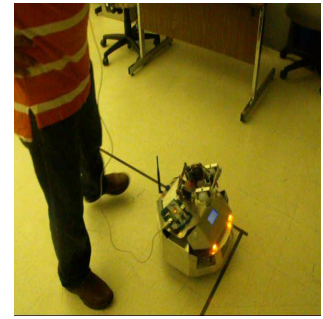

(d)

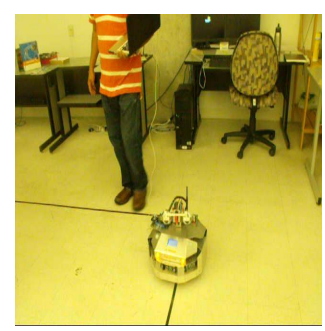

(h)

Figure 10: Robot's real-time performance in tracking a U-shaped trajectory.

environment through an optimal feedback control technique. The proposed controller showed a satisfactory tracking capability despite the deliberately excessive levels of noise and reverberations adopted. Unlike other techniques presented in the literature, the suggested controller does not require linearizing the system in hand, and is modular in the sense that it is applicable to a wide range of control-affine systems. This work can be further extended by taking into account the actuators dynamics, which was not considered here, and incorporating other types of cheap and computationally-simple sensors in conjunction with the RFID system.

\section{References}

[1] R. W. Brockett, Asymptotic stability and feedback stabilization, Differential Geometric Control Theory (1983) 181-191.

[2] Z.-P. Jiang, H. Nijmeijer, Tracking control of mobile robots: a case study in backstepping, Automatica 33 (7) (1997) 1393-9.

[3] Z. P. Jiang, E. Lefeber, H. Nijmeijer, Saturated stabilization and tracking of a nonholonomic mobile robot, Systems \& Control Letters 42 (5) (2001) 327-32. 
[4] T. Fukao, H. Nakagawa, N. Adachi, Adaptive tracking control of a nonholonomic mobile robot, IEEE Transactions on Robotics and Automation 16 (5) (2000) 609-15.

[5] W. E. Dixon, Z. P. Jiang, D. M. Dawson, Global exponential setpoint control of wheeled mobile robots: a lyapunov approach, Automatica 36 (1) (2000) 1741-1746.

[6] T.-C. Lee, K.-T. Song, C.-H. Lee, C.-C. Teng, Tracking control of unicycle-modeled mobile robots using a saturation feedback controller, IEEE Transactions on Control Systems Technology 9 (2) (2001) 305-18.

[7] D. Chwa, Sliding-mode tracking control of nonholonomic wheeled mobile robots in polar coordinates, IEEE Transactions on Control Systems Technology 12 (4) (2004) 637-644.

[8] D. Chwa, Tracking control of differential-drive wheeled mobile robots using a backstepping-like feedback linearization, IEEE Transactions on Systems, Man and Cybernetics, Part A: Systems and Humans 40 (6) (2010) 1285-1295.

[9] P. Morin, C. Samson, Control of nonholonomic mobile robots based on the transverse function approach, IEEE Transactions on Robotics 25 (5) (2009) 1058-73.

[10] D. Sun, C. Wang, W. Shang, G. Feng, A synchronization approach to trajectory tracking of multiple mobile robots while maintaining time-varying formations, IEEE Transactions on Robotics 25 (5) (2009) 1074-1086.

[11] J. H. Lee, C. Lin, H. Lim, J. M. Lee, Sliding mode control for trajectory tracking of mobile robot in the RFID sensor space, International Journal of Control, Automation and System 7 (3) (2009) 429-435.

[12] F. Janabi-Sharifi, M. Marey, A kalman-filter-based method for pose estimation in visual servoing, IEEE Transactions on Robotics 26 (5) (2010) 939-947.

[13] S.-Y. Hwang, J.-B. Song, Monocular vision-based SLAM in indoor environment using corner, lamp, and door features from upward-looking camera, IEEE Transactions on Industrial Electronics 58 (2011) 4804-4812.

[14] C. Siagian, L. Itti, Biologically inspired mobile robot vision localization, IEEE Transactions on Robotics 25 (4) (2009) $861-873$

[15] S. R. Nekoo, Nonlinear closed loop optimal control: A modified state-dependent riccati equation, ISA Transactions 52 (2) (2013) 285-290

[16] S. Park, S. Hashimoto, Autonomous mobile robot navigation using passive RFID in indoor environment, IEEE Transactions on Industrial Electronics 56 (7) (2009) 2366-2373.

[17] V. Kulyukin, C. Gharpure, J. Nicholson, S. Pavithran, RFID in robot-assisted indoor navigation for the visually impaired, in: 2004 IEEE/RSJ IROS, Sendai, Japan, 2004, pp. 1979-84.

[18] D. Hahnel, W. Burgard, D. Fox, K. Fishkin, M. Philipose, Mapping and localization with RFID technology, in: IEEE International Conf. on Robotics and Automation, no. 1, New Orleans, LA, United States, 2004, pp. 10151020 .

[19] B.-S. Choi, J.-W. Lee, J.-J. Lee, K.-T. Park, A hierarchical algorithm for indoor mobile robot localization using RFID sensor fusion, IEEE Transactions on Industrial Electronics 58 (6) (2011) 2226-2235.

[20] S. S. Saad, Z. S. Nakad, A standalone RFID indoor positioning system using passive tags, IEEE Transactions on Industrial Electronics 58 (5) (2011) 1961-1970.

[21] E. D. Giampaolo, F. Martinelli, A passive UHF-RFID system for the localization of an indoor autonomous vehicle, IEEE Transactions on Industrial Electronics 59 (10) (2012) 3961-3970.

[22] G. Kantor, S. Singh, Preliminary results in range-only localization and mapping, in: IEEE ICRA, Washington, DC USA, 2002, pp. 1818-23.

[23] D. Kurth, G. Kantor, S. Singh, Experimental Results in Range-only Localization with Radio, in: IEEE Internationa Conference on Intelligent Robots and Systems, Vol. 1, 2003, pp. 974-979.

[24] W. Gueaieb, S. Miah, An intelligent mobile robot navigation technique using RFID technology, IEEE Transactions on Instrumentation and Measurement 57 (9) (2008) 1908-1917.

[25] M. R. Asharif, B. Moshiri, R. HoseinNezhad, Sensor fusion by pseudo information measure: A mobile robot application, ISA Transactions 41 (3) (2002) 283-301.

[26] L. Teslic, I. Skrjanc, G. Klancar, Using a lrf sensor in the kalman-filtering-based localization of a mobile robot, ISA Transactions 49 (1) (2010) 145-153.

[27] M. Kim, N. Y. Chong, Direction sensing RFID reader for mobile robot navigation, IEEE Transactions on Automation Science and Engineering 6 (1) (2009) 44-54

[28] S. Lanzisera, D. T. Lin, K. S. J. Pister, RF time of flight ranging for wireless sensor network localization, in: Proceedings of the Fourth Workshop on Intelligent Solutions in Embedded Systems, Vienna, Austria, 2006, pp. $165-176$

[29] L. M. Ni, Y. Liu, Y. C. Lau, A. P. Patil, LANDMARC: indoor location sensing using active RFID, in: Proceedings of the First IEEE International Conference on Pervasive Computing and Communications, TX, USA, 2003, pp. $407-15$.

[30] M. Youssef, The horus WLAN location determination system, Ph.D. thesis, University of Maryland, Maryland (2004)

[31] S. Miah, W. Gueaieb, Towards a computationally efficient relative positioning system for indoor environments: An 
RFID approach, in: International Conference on Informatics in Control, Automation and Robotics, Milan, Italy, 2009, pp. 331-336.

[32] H. K. Khalil, Nonlinear Systems, 3rd Edition, Addison-Wesley, Pearson Education Limited,, Upper Saddle River, New Jersey, 2002.

[33] H. L. Royden, P. M. Fitzpatrick, Real Analysis, 4th Edition, Pearson Education Inc., Boston, 2010.

[34] H. Choset, K. M. Lynch, S. Hutchinson, G. Kantor, W. Burgard, L. E. Kavraki, S. Thrun, Principles of Robot Motion: Theory, Algorithms, and Implementations, The MIT Press, Cambridge, Massachusetts, London, England, 2004.

[35] N. U. Ahmed, Elements of Finite-Dimensional Systems and Control Theory, Longman Scientific \& Technical, John Wiley \& Sons, Inc., Essex, England, 1988.

[36] N. U. Ahmed, Dynamic Systems and Control with Applications, World Scientific, New Jersey, 2006.

[37] W. Gueaieb, S. Miah, A modular cost-effective mobile robot navigation system using RFID technology, Journal of Communications 4 (2) (2009) 89-95.

[38] S. Miah, W. Gueaieb, An RFID-based robot navigation system with a customized RFID tag architecture, in: IEEE International Conference on Microelectronics, Cairo, Egypt, 2007, pp. 1658-1663.

[39] A. Bekkali, M. Matsumoto, RFID indoor tracking system based on inter-tags distance measurements, in: Wireless Technology, Vol. 44 of Lecture Notes in Electrical Engineering, Springer US, 2009, pp. 41-62. 